\title{
Autoimmunogenicity during anti-TNF therapy in patients with psoriasis and psoriatic arthritis
}

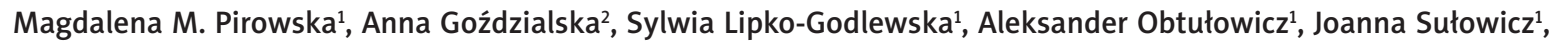 \\ Katarzyna Podolec ${ }^{1}$, Anna Wojas-Pelc ${ }^{1}$
}

${ }^{1}$ Department of Dermatology, Jagiellonian University Medical College, Krakow, Poland Head of the Department: Prof. Anna Wojas-Pelc MD, PhD

${ }^{2}$ Department of Health and Medical Science, Andrzej Frycz Modrzewski Krakow University, Krakow, Poland Head of the Department: Prof. Jerzy Jaśkiewicz MD, PhD

Postep Derm Alergol 2015; XXXII (4): 250-254

DOI: 10.5114/pdia.2015.53320

\begin{abstract}
Introduction: The tumor necrosis factor (TNF- $\alpha$ ) was initially described as lymphotoxin or cachectin. The discovery of therapies blocking the action of TNF- $\alpha$, in 1988, started a new era in the therapy. One of often reported adverse effects related to the use of TNF- $\alpha$ antagonists is induction of the formation of autologous antibodies and antibodies neutralizing anti-TNF drugs. The development of anti-TNF-induced lupus or classical drug-induced lupus is more rarely reported.

Aim: To evaluate the presence and the level of anti-nuclear antibodies in patients with psoriasis and psoriatic arthritis and the influence of anti-TNF therapy used on the concentration of antinuclear antibody (ANA).

Material and methods: A total of 28 subjects were included in the study. $71.4 \%$ of subjects were diagnosed with psoriatic arthritis and $28.6 \%$ with plaque psoriasis.

Results: Among the patients with plaque psoriasis, the antinuclear antibodies were found in $25 \%$ of subjects and in $80 \%$ of patients with psoriatic arthritis. After the treatment an increase in the titer or appearance of antibodies was found in $66.7 \%$ in the infliximab group, $18.2 \%$ in the etanercept group and $54.7 \%$ in the adalimumab group. No subjects developed symptoms of drug-induced systemic lupus.

Conclusions: Our findings have shown that all anti-TNF therapies induced ANA in psoriatic arthritis and psoriatic patients. Considering a mild course of lupus induced by anti-TNF treatment and, usually intrinsic, resolution of symptoms, the biological therapy still appears as a safe treatment for patients.
\end{abstract}

Key words: anti-tumor necrosis factor therapy, psoriasis, psoriatic arthritis, anti-tumor necrosis factor-induced lupus, drug-induced lupus.

\section{Introduction}

The tumor necrosis factor $\alpha$ (TNF- $\alpha)$ was initially described as lymphotoxin or cachectin. It has been suggested that the substance may cause cachexia [1-3]. According to the current state of knowledge, it is one of cytokins, or protein substances participating in inflammatory reaction and immune response of the body. It has been licensed for a variety of inflammatory conditions including psoriasis, psoriatic arthritis, rheumatoid arthritis, ankylosing spondylitis, Crohn's disease, and ulcerative colitis. The discovery of therapies blocking the action of TNF- $\alpha$, in 1988, started a new era in the therapy of the diseases mentioned above. Currently in the European Union, including Poland, three drugs from this group are authorized: infliximab, etanercept and adalimumab for psoriasis and psoriatic arthritis treatment [4].

Infliximab is a recombined, chimeric, mouse-derived, monoclonal antibody against TNF- $\alpha$ cytokine, containing an Fc fragment of human IgG immunoglobulin and variable chains of IgG1k class [5]. Infliximab neutralizes the biological activity of TNF- $\alpha$ by binding the soluble TNF- $\alpha$ (monomers and trimers) and the TNF- $\alpha$ bound with the cell membrane [6]. On the other hand, it does not bind TNF- $\beta$. It shows a capacity for the mediation of the lysis of cells showing the expression of TNF- $\alpha$ (peripheral monocytes, macrophages, neutrophils) by cytotoxicity dependent on the complement or on the antibodies. The drug is administered in an IV infusion, according to

Address for correspondence: Magdalena M. Pirowska MD, PhD, Department of Dermatology, Jagiellonian University Medical College, 8 Skawinska St, 30-066 Krakow, Poland, phone: +48 600858 891, e-mail: pirowska@gmail.com Received: 24.04.2015, accepted: 30.04.2015. 
Summary of Product Characteristics (SPC) in the course of psoriatic arthritis at a dose of $5 \mathrm{mg} / \mathrm{kg}$ b.w. Next time it is administered also at $5 \mathrm{mg} / \mathrm{kg}$ b.w., after 2 and 6 weeks, and then every 8 weeks. This drug is highly effective a reduction of Psoriasis Area Severity Index (PASI) by $75 \%$ (PASI-75) is obtained in about $80 \%$ of patients treated with the recommended dose [4].

Etanercept is a fusion protein consisting of two subunits: a membrane subunit p75 of the TNF- $\alpha$ receptor and a FC part of IgG1 immunoglobulin [5]. It binds two molecules of TNF- $\alpha$, acting as a false receptor. Moreover, it binds TNF- $\beta$ [6]. The drug is administered sub-cutaneously, at a dose of 2 times of $25 \mathrm{mg} /$ week or once, $50 \mathrm{mg} /$ week [4]. The effectiveness of treatment (PASI-75) is evaluated at about $40 \%$ after 12 weeks of therapy. The effectiveness could be increased by using a dose two times larger (i.e. 2 times 50 mg/week) in the first 3 months of therapy. Etanercept is the only biological drug admitted for use in the ordinary psoriasis treatment in children.

Adalimumab is the first fully human monoclonal antibody lgG1k, against the TNF- $\alpha$ cytokine [5]. Adalimumab selectively neutralizes TNF- $\alpha$, inhibiting its binding to p55 and p75 receptors [6]. Adalimumab is administered subcutaneously, with an initial dose of $80 \mathrm{mg}$, then $40 \mathrm{mg}$ a week, and next, 40 mg every 2 weeks in maintenance therapy. The effectiveness of adalimumab in the first 16 weeks of treatment, evaluated with PASI-75 index, is 53-80\%. The treatment with adalimumab allows to reach and maintain PASI-75 in 60\% of patients treated during 60 weeks [4].

The most often reported adverse effects related to the use of TNF- $\alpha$ antagonists are: activation of opportune infections, including Mycobacterium tuberculosis, demyelinating diseases, congestive heart failure, induction of the formation of autologous antibodies and antibodies neutralizing anti-TNF drugs [7]. The development of antiTNF-induced lupus (ATIL) or classical drug-induced lupus (DIL) is more rarely reported.

\section{Aim}

The aim of the work is to evaluate the presence and level of anti-nuclear antibodies in patients with psoriasis and psoriatic arthritis and the influence of anti-TNF therapy used (infliximab, etanercept and adalimumab) on the concentration of antinuclear antibodies (ANA) in patients treated at the Dermatology Clinic of the JagielIonian University Medical College (UJCM).

\section{Material and methods}

A total of 28 subjects were included in the study: 19 men and 9 women, aged 19-67 (mean age 43.7). In the analyzed group, $71.4 \%$ of subjects (20/28) were diagnosed with psoriatic arthritis (according to Caspar criteria) and
28.6\% (8/28) were diagnosed with plaque psoriasis. Six patients were treated with infliximab in a due dose, for 7 months on average. Eleven patients were given etanercept (50 mg s.c./week) during 6-48 months (25.5 on average). Eleven patients were given adalimumab (40 mg s.c./14 days) during 12-36 months (21 on average). The presence and levels of ANA were evaluated before and after treatment. The type of luminescence and the titer were determined by indirect immunofluorescence on Hep-2 cells, using anti-human IgG labeled with fluorescein. Dilutions of 1/160 and higher were considered as positive. The antibodies against soluble antigens: anti-Ro, anti-La, anti-Scl70, anti-Jo1, anti-uRNP, anti-Sm were evaluated by the ELISA method.

\section{Statistical analysis}

The obtained results were processed with statistical analysis.

\section{Results}

In the studied group, the antinuclear antibodies were found, before the inclusion of biological treatment, in $53.6 \%$ of subjects $(15 / 28)$, in the remaining subjects the ANA were not found (46.4\%; $13 / 28$ subjects). Among the patients with positive antibodies, the following titers were observed: $1 / 160$ in 7 (25\%) subjects, $1 / 320$ in 5 (17.9\%), $1 / 640$ in 1 (3.6\%), and 1/1280 in 2 (7.1\%) patients. The distribution of levels of individual antibodies depending on the subjects' sex is presented in Table 1. No significant differences in mean age $(p=1.01)$ and gender $(p=0.73)$ were observed when the patients who became ANA-positive during the treatment were compared to patients who remained persistently ANA-negative.

Among the patients with plaque psoriasis, antinuclear antibodies were found in $25 \%$ of subjects (2/8) and in $80 \%$ of patients with psoriatic arthritis (16/20) (Figure 1 ).

In the group of patients treated with infliximab, the antinuclear antibodies were confirmed in 33.3\% (2/6) before starting the treatment. After the treatment, an increase in the titer or appearance of antibodies was found in $66.7 \%$ (4/6), in one subject the ANA titer decreased. Overall, after the treatment, the ANA were observed in 83.3\% (5/6) (Figure 2).

Table 1. The titer of antibodies depending on the subjects' sex

\begin{tabular}{lccc}
\hline Titer & $\begin{array}{c}\text { Percentage of subjects } \\
(n=28)\end{array}$ & $\begin{array}{c}\text { Percentage } \\
\text { of women }\end{array}$ & $\begin{array}{c}\text { Percentage } \\
\text { of men }\end{array}$ \\
\hline $1 / 160$ & $7 / 28(25 \%)$ & $1 / 28(3.6 \%)$ & $6 / 28(21.4 \%)$ \\
\hline $1 / 320$ & $5 / 28(17.9 \%)$ & $1 / 28(3.6 \%)$ & $4 / 28(14.3 \%)$ \\
\hline $1 / 640$ & $1 / 28(3.6 \%)$ & $1 / 28(3.6 \%)$ & $0 / 28(0 \%)$ \\
\hline $1 / 1280$ & $2 / 28(7.1 \%)$ & $1 / 28(3.6 \%)$ & $1 / 28(3.6 \%)$ \\
\hline
\end{tabular}




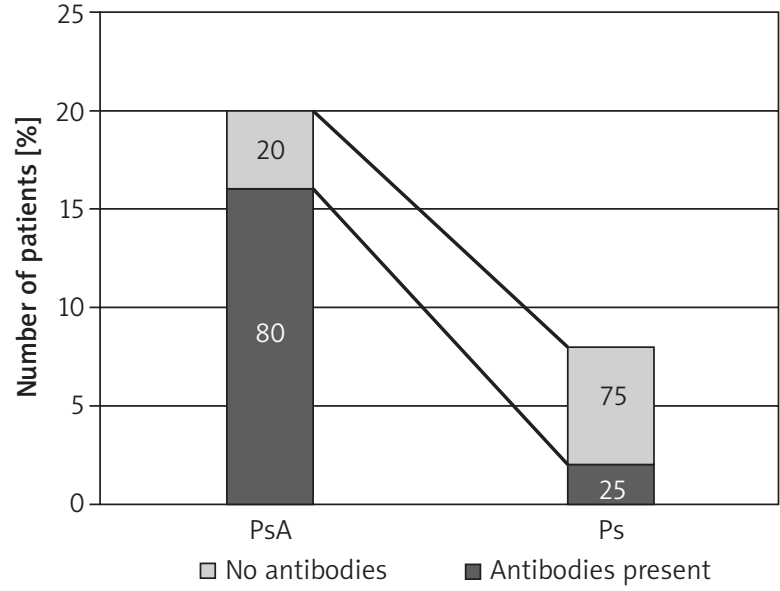

Figure 1. Antinuclear antibodies among the patients with psoriasis and psoriatic arthritis before anti-TNF treatment

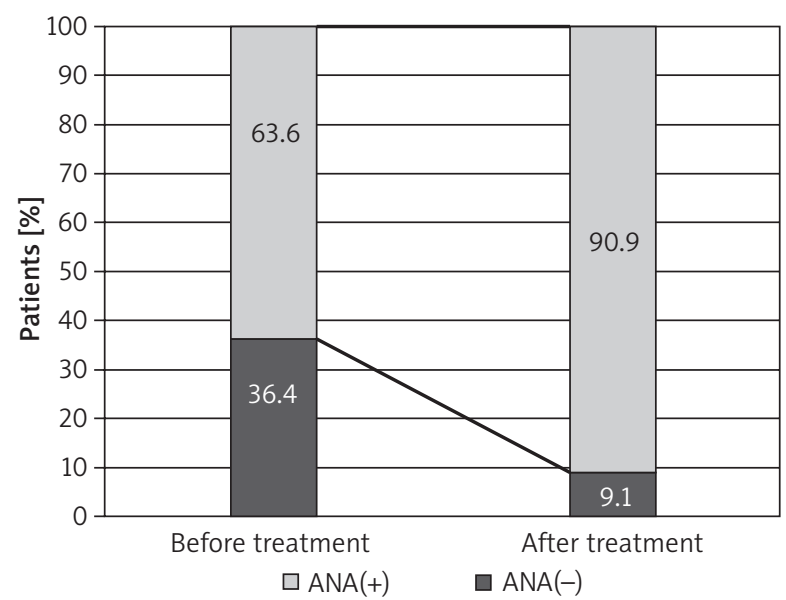

Figure 3. Antinuclear antibodies in the group of patients treated with etanercept

In the group of patients treated with etanercept, the ANA were confirmed in $63.6 \%(7 / 11)$ before starting the treatment. After the treatment, an increase in the titer or appearance of antibodies was found in $18.2 \%$ of subjects. In $63.8 \%$ of patients the level of ANA maintained constant. After the treatment, the ANA were observed in 90.9\% (10/11) (Figure 3).

In the group of patients treated with adalimumab, the ANA were confirmed in 63.6\% (7/11) before starting the treatment. After the treatment, an increase in the titer of ANA was observed in $27.3 \%$ of patients, the generation of antibodies was found in $27.3 \%$ (3/11) of studied subjects. In the remaining patients, the level of antibodies maintained constant. After the treatment, the ANA were observed in 90.9\% (10/11) (Figure 4).

The antibodies against soluble antigens: anti-Ro, anti-La, anti-Scl70, anti-Jo1, anti-uRNP, anti-Sm were not found in any patients.

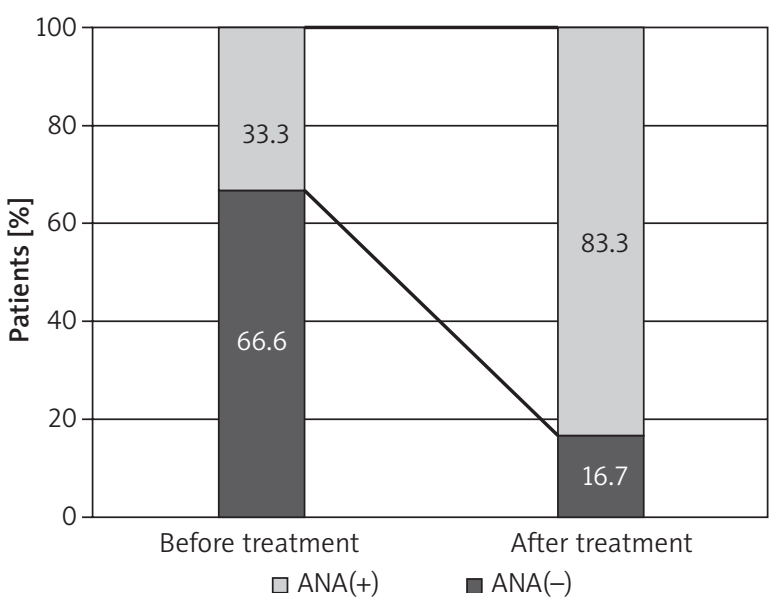

Figure 2. Antinuclear antibodies in the group of patients treated with infliximab

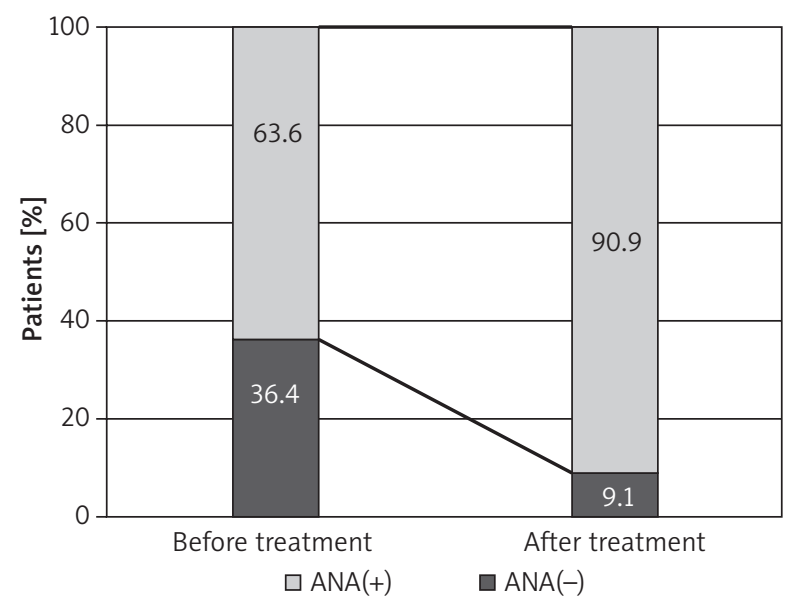

Figure 4. Antinuclear antibodies in the group of patients treated with adalimumab

No subjects developed symptoms of drug-induced systemic lupus.

\section{Discussion}

The first clinical trials with infliximab in the therapy of rheumatoid arthritis have already shown that the formation of ANA during the treatment is possible (ANA in 29-76.7\% of patients, anti-dsDNA in 10-29\%) [8]. In patients treated with other anti-TNF drugs, the frequency of generating antibodies is lower, but also proven. During the therapy with etanercept it is evaluated at $11-36 \%$ and $5-15 \%$, respectively, and with adalimumab $-12.9 \%$ and $5.3 \%$. In the studied group, the percentage of patients with newly generated antibodies against the components of the cell's nucleus was similar [8].

Atzeni et al. claim that the increase in the auto-antibody level after the anti-TNF treatment varies, depend- 
ing on the primary disease, and it is higher in patients with spondyloarthropathy, despite the fact that initially, in non-treated patients, ANA were found more frequently in the group with rheumatoid arthritis [9]. However, the results of other authors do not support these claims [10].

Currently one work documenting the frequency of ANA generation in patients with psoriatic arthritis, treated with anti-TNF drugs, is available in the PubMed base. Among the above patients, the incidence of generation of auto-antibodies is estimated at 47.8\% [11]. Homogenous and granulated patterns dominate. Williams et al. state that new antibodies against the double-stranded DNA are usually generated in IgM and IgA classes, which are considered as non-pathogenic [10]. Anti-dsDNA appear even after a short-term therapy (6 weeks), however, in a long-term observation of patients, no conversion of these antibodies into class IgG, pathognomonic for SLE, was reported [5]. Despite an increase in the antibody level, patients usually respond well to the biological treatment [12]. Lora et al. did not observe differences in psoriasis intensity, measured by PASI scale, in ANA(+) and ANA(-) patients [13]. Nevertheless, some authors report a lower effectiveness of drugs from the anti-TNF group in patients with the presence of anti-dsDNA [14]. Among the patients generating high levels of antibodies against single-stranded DNA (ssDNA) in the IgG class, an increased percentage of therapy interruptions due to allergic skin reactions or general anaphylaxis is reported [15].

It is considered that the generation of autoantibodies during anti-TNF therapy is related to the induction of humoral response due to an inhibition of the response of cytotoxic T-lymphocytes, which in physiological conditions inhibits the reactivity of B-lymphocytes [16]. A hypothesis of the so-called "cytokine shift" is also suggested: the TNF blockers reduce the production of cytokines by Th1 lymphocytes and therefore they shift the immunologic response towards cytokines IL-10 and IFN- $\alpha$, produced by Th2 lymphocytes [17]. Systemic inhibition of the TNF- $\alpha$ activity may affect the apoptosis, weakening the expression of CD44. It leads to a reduction in removing, by phagocytes, the cellular waste (nuclear material) left after apoptosis, and therefore it promotes the production of autoantibodies against DNA and other nuclear antigens [18].

Drug-induced lupus is the most often reported in the therapy with procainamide (20\%) and hydralazine (5$8 \%$ [19]. Among the patients treated with the biological drug, the incidence of anti-TNF-induced lupus is estimated at $0.19 \%$ among the patients receiving infliximab and $0.18 \%$ of patients treated with etanercept [20]. The British Society of Rheumatology (BSR) Biologic Register includes 11394 subjects. In the described group, 40 patients have developed symptoms of lupus induced by anti-TNF therapy (cutaneous or full-blown ATIL). The control group comprises the patients treated with DMARDs (diseasemodifying antirheumatic drugs): lupus was observed in 1 patient [10]. The American researchers note important differences in the antibody profile between patients that developed DIL and ATIL. The classical drug-induced lupus strongly correlates with ANA (> 99\%) and antihistone antibodies (> 95\%); anti-dsDNA are usually absent. The antibody profile of ATIL patients concerns antidsDNA (> 90\%) and anti-histone antibodies (57\%) [10]. Additionally, cutaneous symptoms are much more often observed in ATIL (72\% compared to DIL 9-27\%) [21, 22]. Systemic symptoms typical of systemic lupus occur with a similar frequency in both groups.

Currently there is no standard recommendation as to ATIL prevention. In the literature, single observations on reducing the frequency of the generation of new autoantibodies after including DMARDs in the therapy are available. The preferred treatment for ATIL is the interruption of anti-TNF therapy, which usually leads to the resolution of symptoms [23]. A Spanish group of authors, conducting the research on biological therapies in autoimmune diseases (BIOGEAS), reports a resolution of symptoms of lupus induced by anti-TNF drugs in $94 \%$ of patients. In the studied group, $40 \%$ received glucocorticoids (GCS) treatment, and $12 \%$ received an additional immunosuppression (MTX, LEF, AZA, MMF, CP) [18]. On the other hand, the British Rheumatology Society suggests discontinuing anti-TNF drugs without an additional therapy [19].

Based on the above reports, the researchers pose a question about the safety of anti-TNF treatment and whether it should be continued if it could possibly generate serious autoimmune diseases. There are descriptions of clinical cases in the literature where a transition of systemic lupus erythematosus into rheumatoid arthritis (and vice versa) was found. Sundaramurthy et al. describe 893 patients with lupus, among which 7 have developed rheumatoid arthritis [24]. The coexistence of the above diseases is estimated at $0.09 \%$ of RA cases and is described as a separate disease, "rhupus" [25]. The incidences of "rhupus" and ATIL are similar, which allow for the presumption that some RA patients would have developed SLE independently of the administered therapy [10].

Discontinuation of the therapy with one of anti-TNF drugs due to development of drug-induced lupus (ATIL) does not disqualify the patient from this treatment method. It was noted that after the resolution of lupus symptoms, when a patient treated for Crohn's disease or for rheumatoid arthritis does not meet the diagnostic criteria of lupus erythematosus, inclusion of another drug from this group seems to be safe and possibly effective $[26,27]$.

Considering a mild course of lupus induced by antiTNF treatment and, usually intrinsic, resolution of symptoms, the biological therapy still appears as a safe treatment for patients with severe psoriasis and psoriatic arthritis. 


\section{Conflict of interest}

The authors declare no conflict of interest.

\section{References}

1. Lubecka-Macura A, Kohut M. TNF superfamily - mechanisms of action, biological functions and therapeutic possibilities. Prz Gastroenterol 2010; 5: 303-9.

2. Nedoszytko B, Sokołowska-Wojdyło M, RuckemannDziurdzińska K, et al. Chemokines and cytokines network in the pathogenesis of the inflammatory skin diseases: atopic dermatitis, psoriasis and skin mastocytosis. Postep Derm Alergol 2014; 31: 84-91.

3. Shabgah AG, Fattahi E, Shahneh FZ. Interleukin-17 in human inflammatory diseases. Postep Derm Alergol 2014; 31: 256-61.

4. Szepietowski J, Adamski Z, Chodorowska G, et al. Guidelines of Polish Dermatological Society on the treatment of psoriasis vulgaris and arthropathic psoriasis (psoriatic arthritis) with biological drugs. Przegl Dermatol 2010; 97: 1-13.

5. Kolarz B, Targońska-Stępniak B, Darmochwał-Kolarz D, Majdan M. Autoimmune aspects of treatment with TNF-a inhibitors. Postepy Hig Med Dosw 2007; 61: 478-84.

6. Filipowicz-Sosnowska A. Efficacy and safety of TNF inhibitors - results of randomized controlled clinical trials. Reumatologia 2006; 44, 6: 309-14.

7. Atzeni F, Turiel M, Capsoni F, et al. Autoimmunity and antiTNF-alpha agents. Ann N Y Acad Sci 2005; 1051: 559-69.

8. Atzeni F, Sarzi-Puttini P. Autoantibody production in patients treated with anti-TNF-alpha. Expert Rev Clin Immunol 2008; 4: 275-80.

9. Atzeni F, Talotta R, Salaffi F, et al. Immunogenicity and autoimmunity during anti-TNF therapy. Autoimmun Rev 2013; 12: 703-8.

10. Williams EL, Gadola S, Edwards CJ. Anti-TNF-induced lupus. Rheumatology (Oxford) 2009; 48: 716-20.

11. Viana VS, de Carvalho JF, de Moraes JC, et al. Autoantibodies in patients with psoriatic arthritis on anti-TNF-alpha therapy. Rev Bras Reumatol 2010; 50: 225-34.

12. Eriksson C, Engstrand S, Sundqvist KG, Rantapää-Dahlqvist $\mathrm{S}$. Autoantibody formation in patients with rheumatoid arthritis treated with anti-TNF alpha. Ann Rheum Dis 2005 . 64: 403-7.

13. Aringer M, Steiner G, Graninger WB, et al. Effects of shortterm infliximab therapy on autoantibodies in systemic lupus erythematosus. Arthritis Rheum 2007; 56: 274-9.

14. Lora V, Bonaguri C, Gisondi P, et al. Autoantibody induction and adipokine levels in patients with psoriasis treated with infliximab. Immunol Res 2013; 56: 382-9.

15. Kiss LS, Lovasz BD, Golovics PA, et al. Levels of anti-doublestrained DNA but not antinuclear antibodies are associated with treatment efficacy and adverse outcomes in Crohn's disease patients treated with anti-TNF-alpha. J Gastrointest Liver Dis 2013; 22: 135-40.

16. Comby E, Tanaff P, Mariotte D, et al. Evolution of antinuclear antibodies and clinical patterns in patients with active rheumatoid arthritis with longterm infliximab therapy. J Rheumatol 2006; 33: 24-30.

17. Atzeni F, Turiel M, Capsoni F, et al. Autoimmunity and antiTNF-alpha agents. Ann N Y Acad Sci 2005; 1051: 559-69.

18. Almoallim H, Al-Ghamdi Y, Almaghrabi H, Alyasi O. Anti-tumor necrosis factor-alpha induced systemic lupus erythematosus. Open Rheumatol J 2012; 6: 315-9.

19. Katz U, Zandman-Goddard G. Drug-induced lupus: an update. Autoimmun Rev 2010; 10: 46-50.
20. De Bandt M, Sibilia J, Le Loët X, et al.; Club Rhumatismes et Inflammation. Systemic lupus erythematosus induced by anti-tumour necrosis factor alpha therapy: a French national survey. Arthritis Res Ther 2005; 7: R545-51.

21. Ramos-Casals M, Brito-Zerón P, Muńoz S, et al. Autoimmune diseases induced by TNF-targeted therapies: analysis of 233 cases. Medicine (Baltimore) 2007; 86: 242-51.

22. Mocci G, Marzo M, Papa A, et al. Dermatological adverse reactions during anti-TNF treatments: focus on inflammatory bowel disease. J Crohns Colitis 2013; 7: 769-79.

23. Ledingham J, Deighton C. Update on the British Society for Rheumatology guidelines for prescribing TNF-alpha blockers in adults with rheumatoid arthritis (update of previous guidelines of April 2001). Rheumatology 2005; 44: 157-63.

24. Sundaramurthy SG, Karsevar MP, van Vollenhoven RF. Influence of hormonal events on disease expression in patients with the combination of systemic lupus erythematosus and rheumatoid arthritis. J Clin Rheumatol 1999; 5: 9-16.

25. Panush RS, Edwards NL, Longley S, Webster E. 'Rhupus' syndrome. Arch Intern Med 1988; 148: 1633-6.

26. Santiago T, Santiago MG, Rovisco J, et al. A case of infliximab-induced lupus in a patient with ankylosing spondylitis: is it safe switch to another anti-TNF-alpha agent? Clin Rheumatol 2013; 32: 1819-22.

27. Subramanian S, Yajnik V, Sands BE, et al. Characterization of patients with infliximab-induced lupus erythematosus and outcomes after retreatment with a second anti-TNF agent. Inflamm Bowel Dis 2011; 17: 99-104. 